\title{
Efficiency optimization in small induction motors using magnetic slot wedges
}

\author{
Carlos Verucchi ${ }^{a}$, Cristian Ruschetti $^{\mathrm{a}, *}{ }^{*}$ Esteban Giraldo ${ }^{\mathrm{a}}$, Guillermo Bossio $^{\mathrm{b}}$, José Bossio ${ }^{\mathrm{b}}$ \\ a Grupo INTELYMEC - Universidad Nacional del Centro de la Provincia de Buenos Aires (UNCPBA) and CIFICEN (UNCPBA-CICPBA-CONICET), Olavarría, \\ Buenos Aires, Argentina

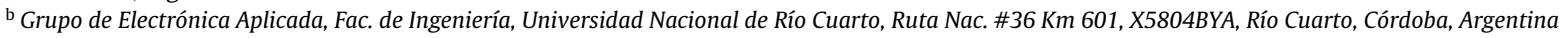

\section{A R T I C L E I N F O}

\section{Article history:}

Received 27 March 2017

Received in revised form 14 June 2017

Accepted 15 June 2017

\section{Keywords:}

Induction motors

Energy efficiency

IEC standards

Magnetic wedges

\begin{abstract}
A B S T R A C T
In the last few years, new efficiency requirements for induction motors have been imposed. Therefore electrical machines manufacturers have had to redefine the design criteria in order to reduce losses aiming to achieve the efficiency values established by the IE2 and IE3 standard categories according to the IEC. Given that, in the near future, an increasing demand regarding efficiency (IE4 and IE5 categories) is expected, it would be appropriate to seek new alternatives to further reduce losses in induction motors.

In this work, the possibility to use magnetic wedges in induction motors with semi-closed slots is studied. This strategy allows to reduce copper and core losses, therefore increasing then the motor efficiency. The study analyses low power motors and considers different permeabilities and geometries for the magnetic wedges. In addition, it focuses attention on the starting torque and currents.

Finally, an experimental validation using a 3-kW 380-V 4-pole IE2-class induction motor is presented.
\end{abstract} (c) 2017 Elsevier B.V. All rights reserved.

\section{Introduction}

The three-phase squirrel-cage induction motors (SCIMs) are present in most industrial processes. Low cost and robustness that characterize SCIMs makes them an almost exclusive alternative for supplying mechanical power. It is estimated that between 80 and $90 \%$ of the total electricity consumed by the industry is used to drive SCIMs [1-3]. Taking into account that approximately 50\% of the electricity generated worldwide is used in the industry, it can be concluded that SCIMs are the major electricity consumers. Therefore reducing losses in these machines becomes a significant objective to be achieved.

For some years, and aligned with changes in the global use of energy resources, new regulations have been incorporated regarding admissible levels of efficiency in SCIMs. Standard efficiency motors, named IE1 by the International Electrotechnical Commission (IEC), are no longer available in the market. Instead, high (IE2) and premium (IE3) efficiency motors [4] are used. In the near future, the incorporation of new efficiency classes to the guidelines set for

\footnotetext{
* Corresponding author.

E-mail addresses:

verucchi@fio.unicen.edu.ar (C. Verucchi), cruschet@fio.unicen.edu.ar (C. Ruschetti), esteban.giraldo@fio.unicen.edu.ar (E.Giraldo), gbossio@ing.unrc.edu.ar (G. Bossio), jbossio@ing.unrc.edu.ar (J. Bossio).
}

induction motors (IE4 and IE5) is expected. Therefore the design criteria to achieve these new levels of efficiency had to be revised [5]. Some possibilities have been tested, with the most important being: the use of magnetic materials of better quality, a more efficient use of the stator slots, the use of efficient bearings and the redesign of the ventilation circuits [6]. All these choices, though capable of reducing losses in SCIMs, do not allow reaching the highest levels of efficiency (IE4, IE5). Some new proposals use line start permanent magnet synchronous motors (LSPMSM) which contains a squirrel cage that allows the startup to be made directly from the electrical grid. This alternative has been tested in up-to-15-kW machines [7-9]. However, the possibility to still improve the efficiency of SCIMs is increasingly limited. Achieving the IE4 efficiency class with random wound windings is considered "difficult". Therefore, it becomes necessary to look for new alternatives in order to reduce losses.

The use of magnetic wedges in the stator slots is widespread within manufactures of medium-voltage induction motors and form wound windings SCIMs [10-16]. Discontinuities in the airgap due to the presence of slots produce harmonic components in the spatial distribution of the magnetic flux. These components produce parasite torques on the motor (and therefore vibrations), add harmonic components to the stator currents, and increase copper losses and stray losses. In low-power machines with random wound windings, semi-closed slots are used in order to improve the spatial flux distribution and minimize its negative effects [17]. On 


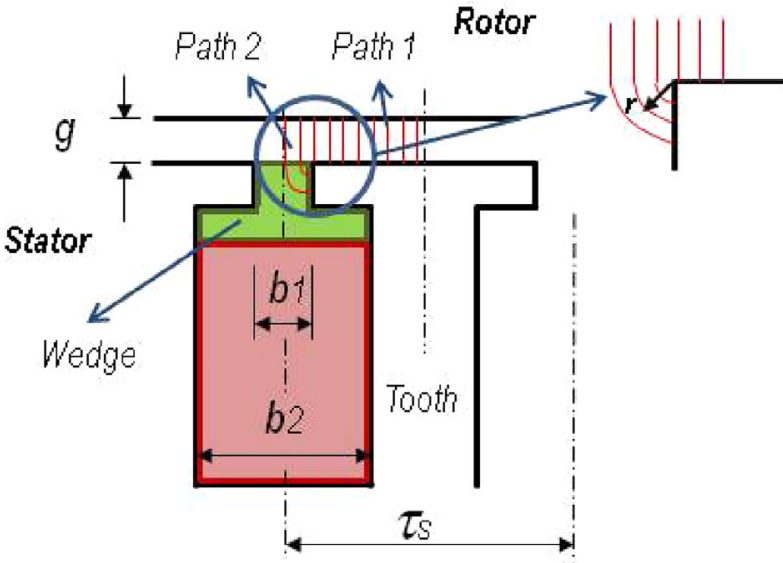

(a)

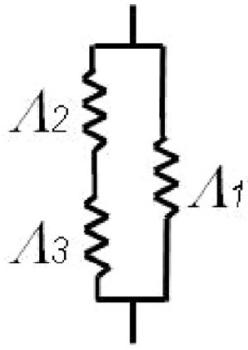

(b)

Fig. 1. Air-gap magnetic permeance in semi-closed slot. (a) Slot and tooth scheme, (b) equivalent circuit.

the contrary, in high-power machines, it is impossible to use semiclosed slots due to the necessity to mount form wound windings. In this case, open slots are used instead and then the presence of parasitic torques becomes a significant problem. A possible solution to this problem is adding magnetic wedges into the stator slots. They reduce the air-gap reluctance differences produced by the slots opening and, therefore, the harmonic components in the magnetic flux. This makes it possible to reduce the air-gap reluctance and the zig-zag magnetic flux, which increase stray losses [18]. This alternative indeed improves efficiency because by reducing the air-gap reluctance, the stator currents are reduced and so are the copper losses. Similarly, increasing the air-gap uniformity reduces the stray losses. Wedges of this type have been mainly associated to medium-voltage and high power motors. In this paper, the possibility to use magnetic wedges in low-voltage motors with semi-closed slots to reduce even more the electrical losses is analyzed.

The literature provides many examples of the advantages of using magnetic wedges in machines with open slots. An analysis of the effects of using magnetic wedges was presented in Ref. [14]. Wedges with different relative permeability values are tested in a $500-\mathrm{kW}$ machine. The results showed that the magnetic wedges reduce the no-load current, the losses associated with the zigzag magnetic flux, those in the magnetic core. However, if the value of the relative permeability of the wedges is indiscriminately increased, undesirable negative effects such as a significant reduction in the starting and the maximum torques of the machine can appear.

In Ref. [15] the performance of a motor with magnetic wedges of different sizes and relative permeability values was analyzed, using the finite element (FE) method. From this analysis, it can be concluded that if certain geometric conditions are not appropriate when placing the magnetic wedges, high-dispersion fluxes can appear in the slots, affecting some operating conditions of the machine such as the power factor.

In Ref. [19], on the other hand, addition to the improvements already mentioned regarding the use of magnetic wedges, it is demonstrated that they are capable of reducing no-load and full load currents in a SCIM improving thus efficiency and the power factor. The works presented in Refs. $[10,12,20]$ reinforce the previous observations on the virtues of using magnetic wedges.

The advantages of using magnetic wedges with a special focus on the economic aspects were also analyzed in Ref. [11]. The highest levels of performance achieved for large motors may result in a significant reduction in energy consumption. A study of a plant with large motors was also presented in this work, as well as the analysis of the energy saving due to the use of magnetic wedges.

Finally, the influence of magnetic wedges on the motor temperature was analyzed in Ref. [21]. A better thermal response of the motor when magnetic wedges are used can be observed. The reduction in the motor temperature is mainly due to the fact that the magnetic wedges avoid smaller concentrations of flux densities in the stator teeth and therefore localized magnetic losses.

There are very few examples in the literature referring to the application of magnetic wedges on induction motors with semiclosed slots. However, the case of an analysis made on a 3-HP, 4 poles, $380 \mathrm{~V}$ SCIM can be mentioned. In this case, it can be concluded that efficiency increases up to $\mu_{r}=20$ and then it starts to decrease. From the same analysis, a reduction in the starting torque and currents can also be observed [22]. In Ref. [23], likewise, the use of magnetic wedges on a $3-\mathrm{kW}, 4$ poles, $380 \mathrm{~V}$ SCIM was studied. This analysis showed encouraging results regarding reduction of the losses.

This paper intends to make a contribution to optimal designs of SCIMs. This proposal especially focuses on reducing losses. With the purpose of meeting this goal, the possibility of improving the performance of a 3-kW SCIM by adding magnetic wedges was analyzed. Firstly, simulations using the FE Method were carried out for wedges with different permeability values and geometries, obtaining for each particular case the performance of the motor at rated speed, the starting current and torque, and the power factor, among other indicators. Finally, an experimental validation was done.

\section{Analysis of the influence of slot wedges on the performance of an induction motor}

\subsection{Magnetizing currents}

In a SCIM, the stator currents needed to magnetize the magnetic core are mainly determined by air-gap magnetic permeance. Fig. 1a shows a simplified view of one stator slot of a SCIM. The magnetic flux goes through the air-gap into two parallel paths. One of these paths corresponds to the region facing a stator tooth, while the other, to the region facing a slot opening. The permeance of the airgap of a half slot pitch is given by the resultant of the series-parallel combination shown in Fig. $1 \mathrm{~b}$. The permeance of path $1\left(\Lambda_{1}\right)$ can be expressed as:

$\Lambda_{1}=\mu_{0} \frac{\tau_{s}-b_{1}}{2} \frac{l_{s}}{g}$ 


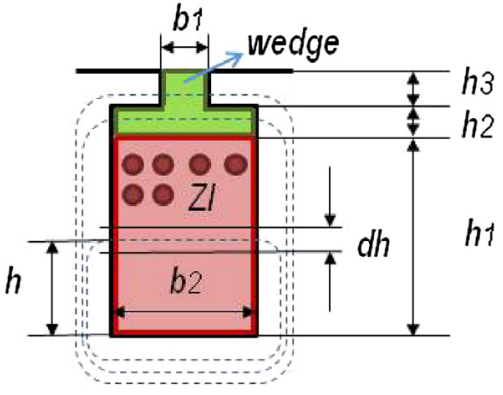

Fig. 2. Flux leakage in semi-closed slot.

where $l_{s}$ is the effective length of the magnetic core. Similarly, for that part of path 2 crossing the air-gap, the permeance is given by:

$\Lambda_{2}=\mu_{0} \frac{b_{1}}{2} \frac{l_{s}}{g}$

Whereas for the one crossing the wedge, the permeance can be obtained as:

$$
d \Lambda_{3}=\mu_{0} \mu_{r} l_{s} \frac{d r}{\pi r / 2} \Rightarrow \Lambda_{3}=\int_{0}^{b_{1} / 2} \frac{2}{\pi} \mu_{0} \mu_{r} l_{s} \frac{d r}{r}=\frac{2}{\pi} \mu_{0} \mu_{r} l_{s} \ln \left(b_{1} / 2\right)
$$

Eq. (3) shows that the magnetic permeance for path 2 increases proportionally with the relative permeability of the wedge $\left(\mu_{r}\right)$, thus permeance along the whole path 2 will be increased by adding magnetic wedges. Permeance at each slot and thus at the air-gap increases as it can be observed in the equivalent circuit of Fig. 1b. Thus, the magnetizing current required to achieve the rated flux at the air-gap will become lower as the relative permeability of the wedge increases. If the permeability of the wedge is in the range of the permeability of the magnetic core material, then the air-gap would not present discontinuities at the slots.

\subsection{Flux leakage}

According to the electrical motor design tradition, leakage inductance $L_{\sigma}$ can be divided into the following partial leakage inductances: air-gap leakage inductance, slot leakage inductance, tooth tip leakage inductance, end winding leakage inductance and skew leakage inductance [24]. Among them, only the slot leakage inductance $L_{u}$ is affected by the presence of a magnetic wedge.

According to the scheme in Fig. 2, $L_{u}$ is represented by the magnetic fluxes closed through the cross sections of the slot indicated as $h_{1}, h_{2}$ and $h_{3}$. Then $L_{u}$ results:

$L_{u}=L_{u 1}+L_{u 2}+L_{u 3}$

It is possible to obtain $L_{u 1}$ by applying Ampere's theorem on the $d h$ section of the slot and assuming infinite permeability for the magnetic core.

$H_{1(h)} b_{2}=Z_{Q} I \frac{h}{h_{1}} \Rightarrow B_{1(h)}=\mu_{0} \frac{Z_{Q} I}{b_{2}} \frac{h}{h_{1}}$

Where, $Z_{Q}$ is the number of turns per slots and $I$, the current at each turn. Then:

$d \lambda_{1(h)}=\mu_{0} \frac{Z_{Q}^{2} I}{b_{2}} \frac{h^{2}}{h_{1}^{2}} l_{e} d h$

$\lambda_{1}=\int_{0}^{h_{1}} \mu_{0} \frac{Z_{Q}^{2} I}{b_{2}} \frac{h^{2}}{h_{1}^{2}} l_{e} d h=\frac{\mu_{0} Z_{Q}^{2} I h_{1} l_{e}}{3 b_{2}}$
Finally, $L_{u 1}$ is:

$L_{u 1}=\mu_{0} \frac{Z_{Q}^{2} h_{1} l_{e}}{3 b_{2}}$

Similarly, for inductances $L_{u 2}$ and $L_{u 3}$ :

$L_{u 2}=\mu_{0} \mu_{r} \frac{Z_{Q}^{2} h_{2} l_{e}}{b_{2}}$

$L_{u 3}=\mu_{0} \mu_{r} \frac{Z_{Q}^{2} h_{3} l_{e}}{b_{1}}$

As it can be observed in Eqs. (9) and (10), the components $L_{u 2}$ and $L_{u 3}$ are affected by the $\mu_{r}$ of the wedges. Therefore it can be concluded that the use of magnetic wedges produces an increase of the machine stator leakage reactance.

\subsection{Induction motor starting characteristics}

An increase in the stator leakage reactance modifies the starting current and torque of a machine. From the SCIM simplified equivalent circuit, it is possible to obtain the following expression for the electromagnetic torque $T_{e}[25]$ :

$T_{e}=\frac{1}{\omega_{s}} \frac{3 R_{r}^{\prime}\left|V_{s}\right|^{2}}{S\left[\left(R_{s}+R_{r}^{\prime} / s\right)^{2}+\left(X_{S}+X_{r}^{\prime}\right)^{2}\right]}$

Where, $R_{r}^{\prime}$ is the rotor resistance referred to the stator, $R_{S}$ is the stator resistance; $X_{S}$ is the leakage reactance of the stator and $X_{r}^{\prime}$ is the leakage reactance of the rotor referred to the stator, and $s$ the slip. Consider a motor 1 , without magnetic wedges and with stator leakage inductance $X_{s 1}$ and a motor 2 , identical to motor 1 , but with magnetic wedges and stator leakage inductance $X_{s 2}$, greater than $X_{S 1}$. Then the starting torques are:

$T_{e 1}=\frac{1}{\omega_{s}} \frac{3 R_{r}^{\prime}\left|V_{s}\right|^{2}}{\left(R_{s}+R_{r}^{\prime}\right)^{2}+\left(X_{s 1}+X_{r}^{\prime}\right)^{2}}$

$T_{e 2}=\frac{1}{\omega_{s}} \frac{3 R_{r}^{\prime}\left|V_{s}\right|^{2}}{\left(R_{S}+R_{r}^{\prime}\right)^{2}+\left(X_{s 2}+X_{r}^{\prime}\right)^{2}}$

Where, $T_{e 1}$ and $T_{e 2}$ are the starting torques for the motor 1 and motor 2 respectively. Dividing both expressions:

$\frac{T_{e 2}}{T_{e 1}}=\frac{\left(R_{s}+R_{r}^{\prime}\right)^{2}+\left(X_{s 1}+X_{r}^{\prime}\right)^{2}}{\left(R_{s}+R_{r}^{\prime}\right)^{2}+\left(X_{s 2}+X_{r}^{\prime}\right)^{2}}=k$

Where, the $\mathrm{k}$-factor less than 1 indicates that the starting torque after adding the magnetic wedges $\left(T_{e 2}\right)$ is lower than the original starting torque of the motor without wedges $\left(T_{e 1}\right)$.

The stator current at the starting instant can be calculated as:

$I_{S}=\frac{\left|V_{s}\right|}{\sqrt{\left(R_{S}+R_{r}^{\prime}\right)^{2}+\left(X_{S}+X_{r}^{\prime}\right)^{2}}}$

Repeating the above procedure, it can be concluded that:

$\frac{I_{s 2}}{I_{s 1}}=\frac{\sqrt{\left(R_{s}+R_{r}^{\prime}\right)^{2}+\left(X_{s 1}+X_{r}^{\prime}\right)^{2}}}{\sqrt{\left(R_{s}+R_{r}^{\prime}\right)^{2}+\left(X_{s 2}+X_{r}^{\prime}\right)^{2}}}=\sqrt{k}$

Where, $I_{s 1}$ and $I_{s 2}$ are the starting currents for motor 1 and motor 2 respectively. From Eqs. (14) and (16) it can be concluded that the use of magnetic wedges produces a higher reduction in the starting torque than in the starting current. Fig. 3 shows the variations of the starting torque and the starting current of a $3-\mathrm{kW}$ motor in relation to its original values and for different values of the stator leakage reactance.

Based on the above considerations, it can be stated that the magnetic wedges can reduce losses in a semi-closed-slot induction motor. However, this solution increases the stator leakage 


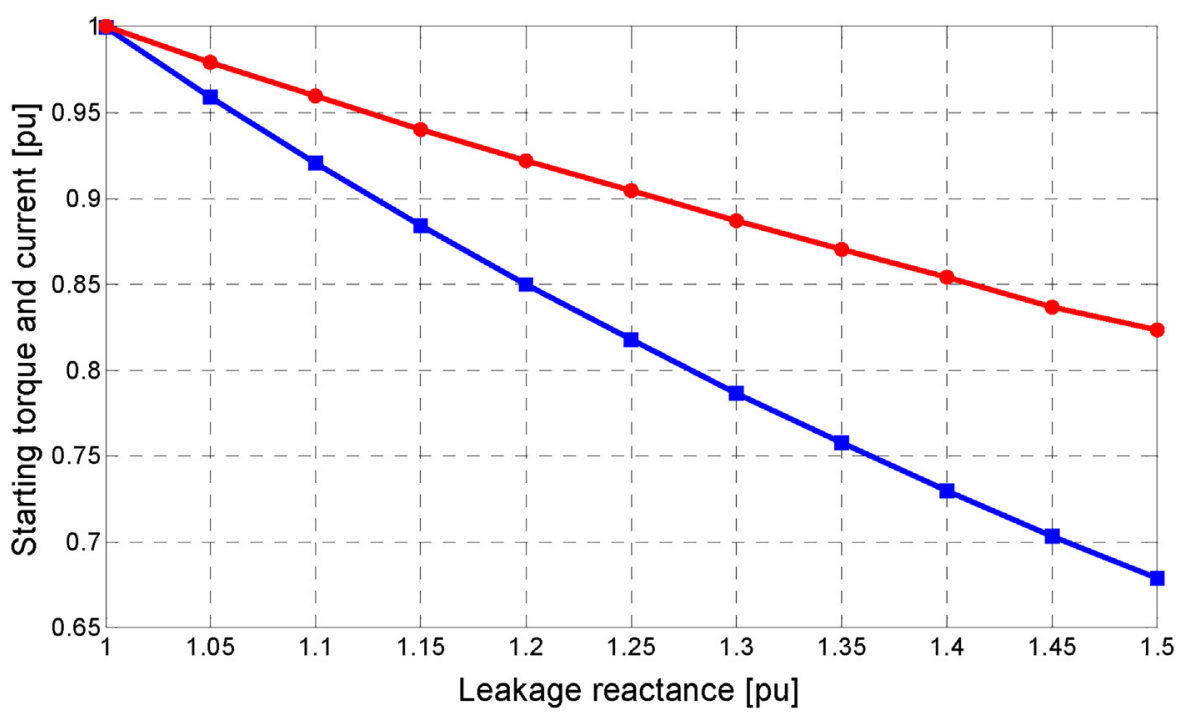

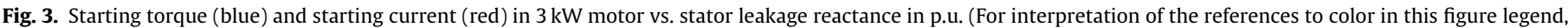
the reader is referred to the web version of this article.)

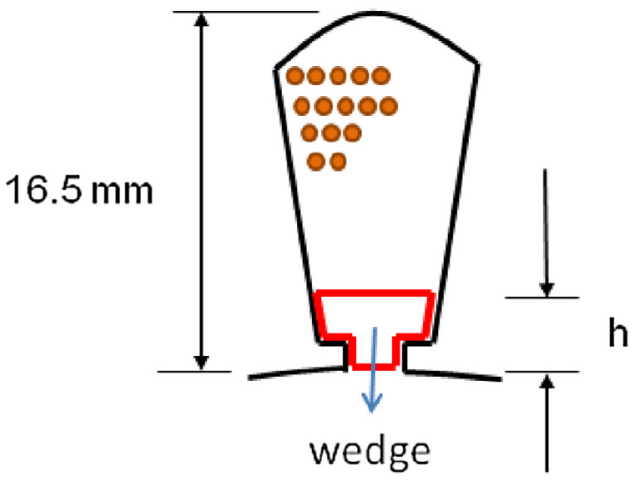

Fig. 4. Semi-closed slot and wedge.

reactance. This increase in turn reduces the starting current and decreases the starting torque of the motor even more. Such conclusions assume the linear behavior of the motor magnetic circuit. During the motor startup, the stator currents take high values. This generally causes magnetic core saturation. Then, the starting performance of the motor may not be affected as much as stated along with the linear model. For definitive conclusions, FE simulations are presented in the following sections as well as an experimental validation.

\section{Finite element $2 D$ numerical analysis}

The proposal presented in this paper is aimed at low-power SCIMs and it was tested in a $3-\mathrm{kW}, 380 \mathrm{~V}, 4$ poles, IE2-class motor. These machines are usually constructed with wooden wedges or wedges made of insulating materials, especially designed to hold the coils within the slots. From a 2D model, the behavior of the motor under no-load and full load conditions with wedges of different permeability and dimensions was simulated using FE Method.

Fig. 4 shows a scheme of a motor slot with a magnetic wedge. The design of the wedges (rectangular and fitting the slot openings) aims at homogenizing the air-gap discontinuity due to the presence of slots while making their construction easier. In fact, the magnetic material used to build the wedges is usually available in plates of different thickness from which the pieces for each motor are obtained. In Fig. 5 the $B-H$ characteristic curves of different materials used on the magnetic wedges are shown. From these data,

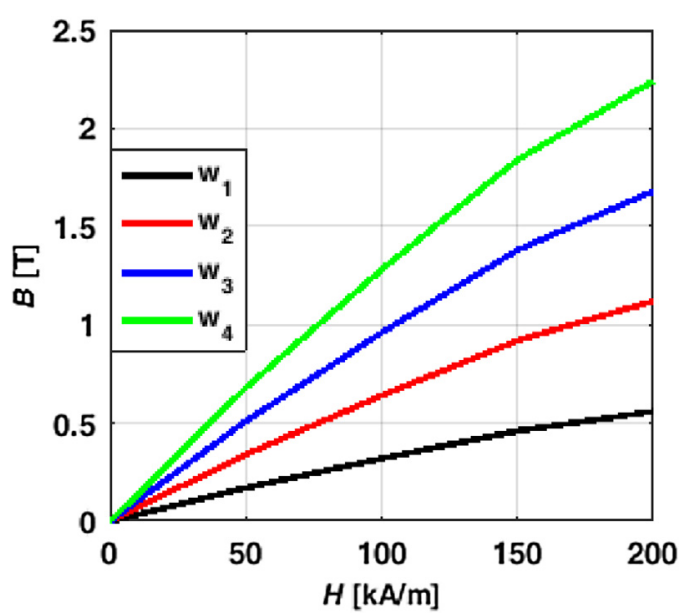

Fig. 5. $B-H$ characteristic curves for different magnetic wedge materials.

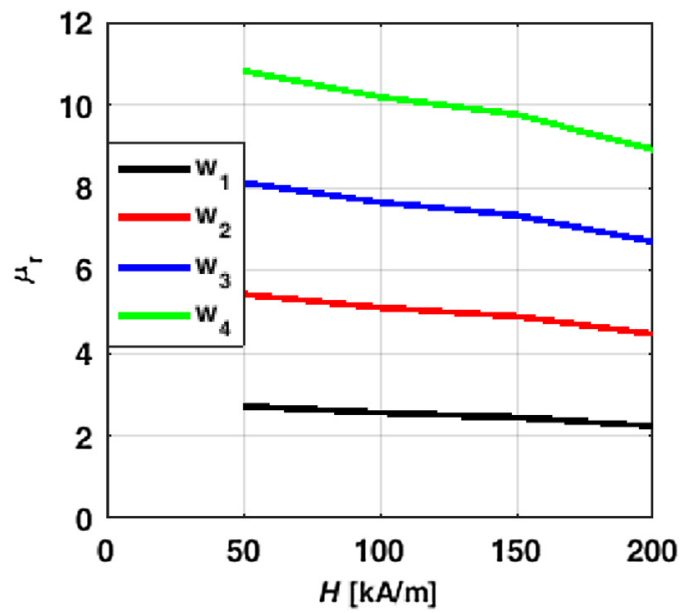

Fig. 6. Relative permeability vs. $H$, for different magnetic wedge materials.

the value of relative permeability is calculated for each material, as shown in Fig. 6. With the obtained values was proceeded to identify each material with an approximate mean permeability value 
Table 1

Comparison of simulation results (permeability).

\begin{tabular}{|c|c|c|c|c|c|}
\hline Performance $(\mathrm{h}=2.5 \mathrm{~mm})$ & $\mu_{r}=1$ & $\mu_{r}=2.5$ & $\mu_{r}=5$ & $\mu_{r}=8$ & $\mu_{r}=10$ \\
\hline No load current (A) & 4.1 & 3.85 & 3.64 & 3.51 & 3.44 \\
\hline Full load current (A) & 6.66 & 6.54 & 6.47 & 6.46 & 6.46 \\
\hline Power factor & 0.80 & 0.80 & 0.80 & 0.81 & 0.81 \\
\hline Torque at starting (Med) (Nm) & 77.3 & 75.45 & 71.7 & 68.72 & 65.42 \\
\hline Stator copper loss (W) & 193 & 186 & 182 & 182 & 182 \\
\hline Rotor copper loss (W) & 108 & 100 & 98 & 100 & 101 \\
\hline Iron loss $(\mathrm{W})$ & 114 & 108 & 104 & 104 & 104 \\
\hline Mechanical loss (W) & 11.1 & 11.2 & 11.2 & 11.1 & 11.1 \\
\hline Stray loss (W) & 57 & 46 & 29 & 28 & 32 \\
\hline Efficiency (\%) & 87.63 & 88.37 & 89.07 & 88.96 & 88.78 \\
\hline
\end{tabular}

that allows making a more simple description during the development of this work. The relative permeability values considered to each wedge are: $\mu_{r}=1$ (no-magnetic material wedge), $\mu_{\mathrm{r}}=2.5$ to wedge $\mathrm{w}_{1}, \mu_{r}=5$ to wedge $\mathrm{w}_{2}, \mu_{r}=8$ to wedge $\mathrm{w}_{3}$ and finally $\mu_{r}=10$ to wedge $w_{4}$. Regarding the wedge heights ( $h$ in Fig. 4 ), the following values were considered: $2,2.5$ and $3 \mathrm{~mm}$. These numbers were chosen as functions of the slot openings. It requires a slight modification of the filling factor.

In order to calculate the magnetic losses, the machine materials data provided by the manufacturer and the magnetic losses characterization of the material had been taken into account to the simulation model. The material corresponds to non-oriented electrical steel M530-50. The effect of the temperature on the magnetic losses has not been taken into account when modeling the machine, but the material characterization losses correspond to the machine material in work conditions. However, recent studies show that hysteresis and eddy current losses hardly decrease with the temperature in the operating temperature range of the industrial electrical machines [26,27]. All other parameters, like copper resistivity, used in the model were obtained of the prototype at the testing temperature.

The magnetic wedge material is the same that is used to build medium voltage electrical machines. These wedges are of a glass fiber as carrier, iron powder and epoxy resin as a binding agent. Its use is widely disseminated and tested. The electrical resistivity of the magnetic wedge material is very high and therefore the effect of the eddy current losses in the wedge is negligible. In addition, an increase in the temperature produces an increase in the electrical resistivity value and, therefore, the effect of eddy losses is less appreciable. Therefore, the magnetic losses on the wedges are neglected.

\subsection{Finite element results}

Table 1 shows the results obtained for the main motor variables. For this particular case, the height of the wedge is $2.5 \mathrm{~mm}$ and the relative permeability values vary between 1 and 10. From Table 1 , it can be concluded that an increase in permeability causes a reduction in the no-load current of up to about $16 \%$. Regarding the rated current, it presents a reduction initially and then reaches a minimum top value for $\mu_{r}=5$. The power factor does not show significant variations. The average starting torque shows a reduction of $2.4 \%$ for $\mu_{r}=2.5$ wedges (the average starting torque was defined as the mean value between the standby position and until the rotor reaches the no-load speed). This drop is even more pronounced for grater permeability values. Regarding losses, it can be observed that the stator copper losses show a higher reduction with the increase in permeability. On the contrary, iron and stray losses show a slight reduction. The motor efficiency finally increases up to $\mu_{r}=8$ and then it starts decreasing. This behavior can be explained by the fact that above a certain $\mu_{r}$ value, the stator copper losses
Table 2

Comparison of simulation results (height).

\begin{tabular}{llll}
\hline Performance $\left(\mu_{r}=5\right)$ & $\mathrm{h}=2 \mathrm{~mm}$ & $\mathrm{~h}=2.5 \mathrm{~mm}$ & $\mathrm{~h}=3 \mathrm{~mm}$ \\
\hline No load current (A) & 3.69 & 3.64 & 3.59 \\
Full load current (A) & 6.47 & 6.47 & 6.48 \\
Power factor & 0.81 & 0.80 & 0.80 \\
Torque at starting (Med) (Nm) & 72.68 & 71.7 & 70.1 \\
Stator copper loss (W) & 182 & 182 & 183 \\
Rotor copper loss (W) & 97.5 & 98 & 95.5 \\
Iron loss (W) & 105 & 104 & 104 \\
Mechanical loss (W) & 11.1 & 11.2 & 11.2 \\
Stray loss (W) & 31 & 29 & 33 \\
Efficiency (\%) & 88.99 & 89.07 & 88.97 \\
\hline
\end{tabular}

Table 3

Motor specification.

\begin{tabular}{ll}
\hline Rated power & $3 \mathrm{~kW}$ \\
Number of poles & 4 \\
Rated voltage & $380 \mathrm{~V}$ \\
Rated frequency & $50 \mathrm{~Hz}$ \\
Rated current & $6.6 \mathrm{~A}$ \\
Rated speed & $1430 \mathrm{RPM}$ \\
Stator slots number & 36 \\
Size & $100 \mathrm{~L}$ \\
IEC efficiency & IE2 \\
\hline
\end{tabular}

stop decreasing. Small variations of the stray losses produce indeed performance deterioration of the motor.

Table 2 shows a comparison of the behavior of the motor variables for fixed permeance and variable heights of the wedges. Like what happens with permeance, an increase in the wedge height produces initially an increase in performance; then, above a certain height value, it starts decreasing. Then, it can be concluded that the influence of the wedge height on the motor variables is lower than that of its $\mu_{r}$.

Fig. 7 shows the efficiency values obtained at rated speed. Taller wedges are able to achieve better performance. For each case, there is an optimum permeability value, above which the efficiency begins to decrease. The efficiency of the original motor, obtained from simulation, is $87.63 \%$ (manufacturers suggest $87.5 \%$ ). The best results are obtained for $3 \mathrm{~mm}$-height wedges with $\mu_{r}=8$. In this particular case, efficiency reaches $89.13 \%$, that is, $2.36 \%$ higher than the original value. The mean starting torque reduces from $77.28 \mathrm{Nm}$ to $64.45 \mathrm{Nm}$ (16\%).

Figs. 8 and 9 present efficiency and starting torque results for different permeability and height values.

\section{Experimental results}

In order to experimentally validate the conclusions stated above, the original wedges were replaced by 2.5 -mm-height magnetic wedges with relative permeability $\mu_{r}=2.5$ in a $3-\mathrm{kW}$ SCIM. Table 3 displays the original characteristics of the motor. The wedges were shaped out of a $2.5-\mathrm{mm}$-thick plate (see mounting wedges in 


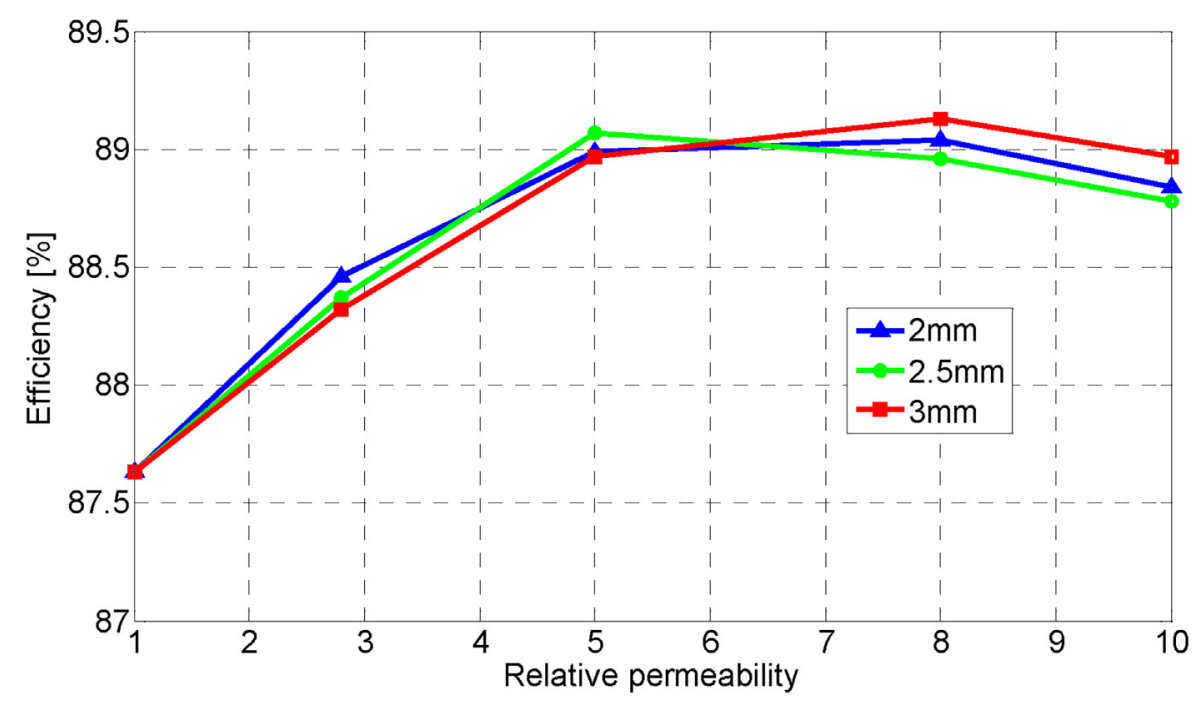

Fig. 7. Efficiency versus permeability at different heights wedges.

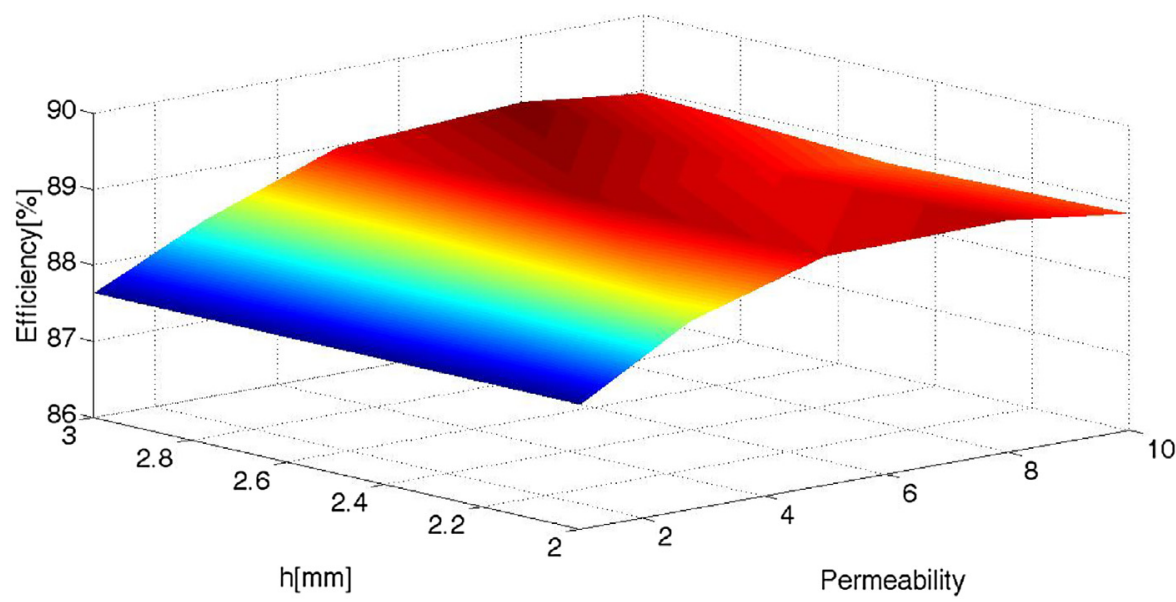

Fig. 8. Efficiency versus permeability and height wedge.

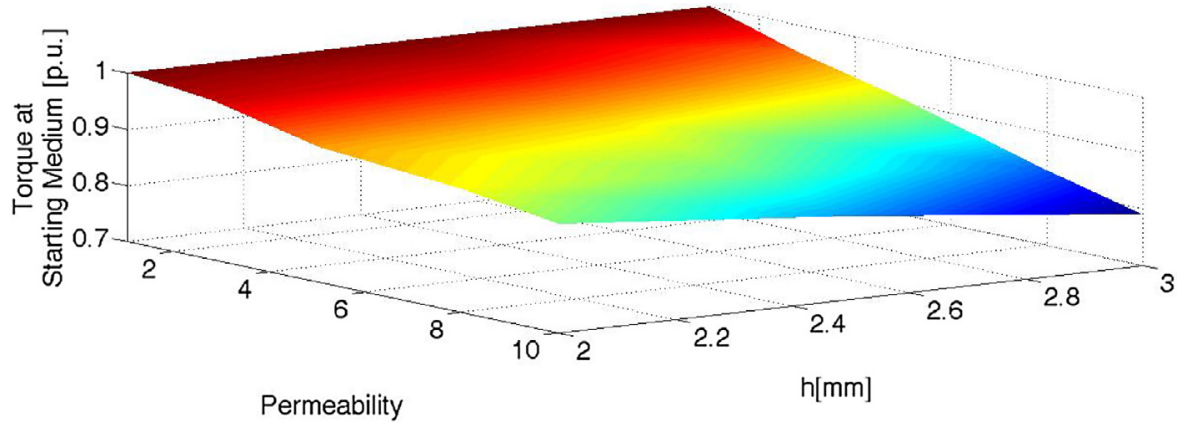

Fig. 9. Starting torque (medium value) versus permeability and height wedge.

Fig. 10) and according to the geometry shown in Fig. 4. Tests were carried out by applying the so called direct method for efficiency measurement [28].

Table 4 presents a comparison of the different variables of the motor for original and magnetic wedges.

Experimental results show small differences compared with those obtained from simulation using the FE Method. Regarding efficiency, the values obtained experimentally were slightly lower than expected. This is mainly due to the fact that it was not possible to avoid small voltage imbalances or harmonic components exper-
Table 4

Experimental results.

\begin{tabular}{llll}
\hline & $\mu_{r}=1$ & $\mu_{r}=2.5$ & $\Delta(\%)$ \\
\hline Efficiency & 86 & 87.2 & 1.4 \\
Starting current & 56.1 & 52.7 & -6 \\
Starting torque & 68 & 64 & -5.9 \\
\hline
\end{tabular}

imentally. However, trends are clear and it can be assured that the conclusions obtained from simulations are reliable. 


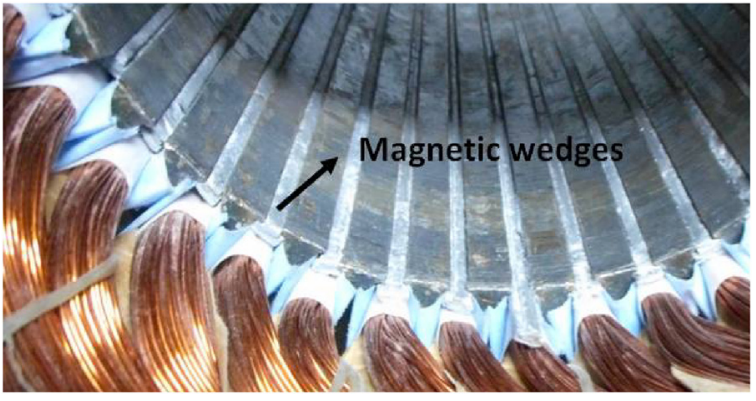

Fig. 10. Replacement of wedges in 3-kW induction motor.

The comparison presented in Table 4 shows that it is possible to improve the motor efficiency by means of using magnetic wedges. What's more, higher efficiency values might be obtained by reducing the starting torque.

\section{Discussion}

One of the biggest challenges that manufacturers face currently is to improve motor efficiency. It is yet difficult to reach the efficiency values established by the standard categories IE4 and IE5 (not available) [4]. Some experts believe that in many cases such efficiency classes can hardly be reached with SCIMs and propose alternatives such as LSPMSM or Synchronous Reluctance Motor (SynRM) [29]. The proposal presented in this paper puts into consideration a new possibility to further reduce losses in SCIMs.

The starting torque reduction that SCIMs undergo when using magnetic wedges is not significant. For the case analyzed in the present work, the starting torque showed a reduction of about $6 \%$, which means that it is 2.6 times the motor rated torque. For all the cases analyzed by means of computer simulations, the reached starting torques made the motors keep their original category in terms of starting conditions, IEC Design $\mathrm{N}$ in this case [30]. A reduction of the starting current is an additional improvement for the motor. Similarly and as it is shown in Ref. [31], an increase of the stator reactance implies a better response of the motor for unbalances or the presence of harmonic components in the power source.

Another interesting point of the proposal lies in its economic aspects. Although so far it has not been possible to establish the increase of costs for each unit when using magnetic wedges, it could be ensured that it would be below the additional costs other alternatives present (a IE4-class LSPMSM shows costs about 233\% higher that a IE2- class SCIM [5]). Unlike open-slot motors, in which there are frequent failures during the mounting process of magnetic wedges due to the electrodynamic stress they undergo [32], in semi-closed slot machines, the geometry of the slot and wedge allows a safer mounting.

\section{Conclusions}

A design option for an optimal design of SCIMs has been presented in this paper. The proposal focuses on reducing losses and increasing motor efficiency. Numerical analysis to evaluate different cases in relation to the relative permeability of the wedges and their dimension were carried out. Finally, experimental validations reinforce conclusions.

\section{Acknowledgments}

This work was supported by the Universidad Nacional del Centro de la Provincia de Buenos Aires, by the Universidad Nacional de
Río Cuarto, by FONCyT-ANPCyT and by the Consejo Nacional de Investigaciones Científicas y Técnicas (CONICET).

\section{References}

[1] R. Saidur, A review on electrical motors energy use and energy saving, Renew. Sustain. Energy Rev. 14 (2010) 877-898.

[2] C. Verucchi, C. Ruschetti, G. Kazlauskas, High efficiency electric motors: economic and energy advantages, IEEE Lat. Am. Trans. 11 (6) (2013) 1326-1332.

[3] P. Gnacinski, T. Tarasiuk, Energy-efficient operation of induction motors and power quality standards, Electr. Power Syst. Res. 135 (2016) 10-17.

[4] Rotating Electrical Machines-Part 30-1: Efficiency classes of line operated AC motors (IE code), IEC 60034-30-1, first ed., 2014.

[5] A.T. de Almeida, F.J.T.E. Ferreira, B. Ge, Beyond induction motors-technology trends to move up efficiency, IEEE Trans. Ind. Appl. 50 (3) (2014) 2103-2141.

[6] C. Yung, Tips for improving motor efficiency, IEEE Ind. Appl. Mag. 12 (50) (2007) 12-20.

[7] M. Melfi, S. Evon, R. Mcelveen, Induction versus permanent magnet motor, IEEE Ind. Appl. Mag. (2009) 28-35.

[8] R.T. Ugale, B.N. Chaudhari, Rotor configurations for improved starting and synchronous performance of line start permanent magnet synchronous motor, IEEE Trans. Ind. Electron. 64 (1) (2017) 138-148.

[9] J.J. Lee, S.H. Rhyu, I.S. Jung, Y.W. Kim, Design of high efficiency line start permanent magnet motor for submersible pumps, in: IEEE 16th International Conference on Environment and Electrical Engineering (EEEIC), Florence, Italy, 2016, pp. 1-4.

[10] S. Wang, Z. Zhao, L. Yuan, B. Wang, Investigation and analysis of the influence of magnetic wedges on high voltage motors performance, in: IEEE Vehicle Power and Propulsion Conference, VPPC ‘08, Harbin, China, 2008, pp. 1-6.

[11] R. Curiac, H. Li, Improvements in energy efficiency of induction motors by the use of magnetic wedges, Conf. Rec. IEEE IAS Annu. Meeting PCIC (2011) 1-6.

[12] M. Skalka, C. Ondrusek, J. Kurfurst, R. Cipin, Harmonic reduction in induction machine using slot wedges optimization, International Symposium on Power Electronics, Electrical Drives, Automation and Motion (SPEEDAM) (2012) 1252-1255.

[13] J. Kappatou, C. Gyftakis, A. Safacas, A study of the effects of the stator slots wedges material on the behavior of an induction machine, 18th International Conference on Electrical Machines, ICEM 2008 (2008) 1-6.

[14] Y. Takeda, T. Yagisawa, A. Suyama, M. Yamamoto, Application of magnetic wedges to large motors, IEEE Trans. Magn. 20 (1984) 1780-1782.

[15] H. Mikami, K. Ide, M. Takahashi, K. Kajiwara, Dynamic harmonic field analysis of a cage type induction motor when magnetic slot wedges are applied, IEEE Trans. Energy Convers. 12 (1997) 337-343.

[16] R. Curiac, H. Li, Improvements in energy efficiency of induction motors by the use of magnetic wedges, Petroleum and Chemical Industry Conference (PCIC) Record of Conference Papers Industry Applications Society 58th Annual IEEE (2011) 1-6.

[17] J. Le Besnerais, V. Lanfranchi, M. Hecquet, R. Romary, P. Brochet, Optimal slot opening width for magnetic noise reduction in induction motors, IEEE Trans. Energy Convers. 44 (4) (2009) 869-874.

[18] Y. Anazawa, A. Kaga, H. Akagami, S. Watabe, M. Makino, Prevention of harmonic torques in squirrel cage induction motors by means of soft ferrite magnetic wedges, IEEE Trans. Magn. 18 (6) (1982) 1550-1552.

[19] G. Madescu, M. Greconici, M. Biriescu, M. Mot, Effects of stator slot magnetic wedges on the induction motor performances, 13th International Conference on optimization of Electrical and Equipment (OPTIM) (2012) 489-492.

[20] K.N. Gyftakis, P.A. Panagiotou, J. Kappatou, The influence of semi-magnetic wedges on the electromagnetic variables and the harmonic content in induction motors, XXth International Conference on Electrical Machines, ICEM 2012 (2012) 1469-1474.

[21] T. Gaerke, D. Hernandez, The temperature impact of magnetic wedges on TEFC induction motors, IEEE Trans. Ind. Appl. 49 (3) (2013) 1228-1233.

[22] M. Lavanya, P. Selvakumar, S. Vijayshankar, C. Easwarlal, Performance analysis of three phase induction motor using different slot wedges, IEEE 2nd International Conference on Electrical Systems (ICEES) (2014) 164-167.

[23] E. Giraldo, C. Ruschetti, C. Verucchi, G. Bossio, J. Bossio, Optimización de la eficiencia en motores de inducción de ranuras semiabiertas mediante empleo de cuñas magnéticas, in: IEEE Biennial Congress of Argentina (ARGENCON 16), Buenos Aires, Argentina, 2016, pp. 1-6.

[24] J. Pyrhönen, T. Jokinen, V. Hrabovcová, Design of Rotating Electrical Machines, John Wiley \& Sons, Ltd, Great Britain, 2008, pp. 227-253.

[25] A.E. Fitzgerald, C. Kingsley, D. Umans, Electric Machinery, sixth ed., McGraw-Hill Higher Education, New York, USA, 2003, pp. 313-317.

[26] J. Chen, Z. Chen, D. Wang, L. Wu, X. Zheng, F. Birnkammer, D. Gerling, Influence of temperature on magnetic properties of silicon steel lamination, AIP Adv. 7 (5) (2017)

[27] S. Hussain, V. Ghorbanian, A. Benabou, S. Clénet, D.A. Lowther, A study of the effects of temperature on magnetic and copper losses in electrical machines, in: 2016 XXII International Conference on Electrical Machines (ICEM), Lausanne, 2016, pp. 1277-1283.

[28] G. Bucci, F. Ciancetta, E. Fiorucci, A. Ometto, Uncertainty issues in direct and indirect efficiency determination for three-phase induction motors: remarks about the IEC 60034-2-1 standard, IEEE Trans. Instrum. Meas. 65 (12) (2016) 2701-2716. 
[29] A. De Almeida, F. Ferreira, A. Duarte, Technical and economical considerations on super high-efficiency three-phase motors, IEEE Trans. Ind. Appl. 50 (2) (2014) 1274-1285.

[30] Rotating Electrical Machines-Part 12: Starting performance of single-speed three-phase cage induction motors, IEC 60034-12, second ed., 2002.

[31] P.D. Donolo, G.R. Bossio, C. De Angelo, Analysis of voltage unbalance effects on induction motors with open and closed slot, Energy Convers. Manag. 52 (5) (2011) 2024-2030.
[32] G. Bossio, P. De La Barrera, J. Bossio, C. Verucchi, R. Leidhold, Fault detection in magnetic wedges of induction motor, IEEE 24th IEEE International Symposium on Industrial Electronics, ISIE 2015 (2015) 506-511. 